OPEN ACCESS

Edited by:

Christian Lohr.

University of Hamburg, Germany

Reviewed by:

Lynne Ann Oland,

University of Arizona, United States

Kevin C. Daly,

West Virginia University, United States

*Correspondence:

Xin-Cheng Zhao xincheng@henau.edu.cn

Received: 07 September 2017 Accepted: 08 November 2017

Published: 21 November 2017

Citation:

Ma B-W, Zhao X-C, Berg BG, Xie G-Y, Tang $Q-B$ and Wang $G-R$ (2017) Central Projections of Antennal and Labial Palp Sensory Neurons in the Migratory Armyworm Mythimna separata.

Front. Cell. Neurosci. 11:370. doi: 10.3389/fncel.2017.00370

\section{Central Projections of Antennal and Labial Palp Sensory Neurons in the Migratory Armyworm Mythimna separata}

\author{
Bai-Wei Ma' ${ }^{1}$ Xin-Cheng Zhao ${ }^{1 *}$, Bente G. Berg ${ }^{2}$, Gui-Ying Xie ${ }^{3}$, Qing-Bo Tang ${ }^{1}$ and \\ Gui-Rong Wang ${ }^{4}$
}

' Department of Entomology, College of Plant Protection, Henan Agricultural University, Zhengzhou, China, ${ }^{2}$ Department of Psychology, Norwegian University of Science and Technology, Trondheim, Norway, ${ }^{3}$ Department of Pesticide, College of Plant Protection, Henan Agricultural University, Zhengzhou, China, ${ }^{4}$ State Key Laboratory for Biology of Plant Disease and Insect Pests, Institute of Plant Protection, Chinese Academy of Agricultural Sciences, Beijing, China

The oriental armyworm, Mythimna separata (Walker), is a polyphagous, migratory pest relying on olfactory cues to find mates, locate nectar, and guide long-distance flight behavior. In the present study, a combination of neuroanatomical techniques were utilized on this species, including backfills, confocal microscopy, and three-dimensional reconstructions, to trace the central projections of sensory neurons from the antenna and the labial pit organ, respectively. As previously shown, the axons of the labial sensory neurons project via the ipsilateral labial nerve and terminate in three main areas of the central nervous system: (1) the labial-palp pit organ glomerulus of each antennal lobe, (2) the gnathal ganglion, and (3) the prothoracic ganglion of the ventral nerve cord. Similarly, the antennal sensory axons project to multiple areas of the central nervous system. The ipsilateral antennal nerve targets mainly the antennal lobe, the antennal mechanosensory and motor center, and the prothoracic and mesothoracic ganglia. Specific staining experiments including dye application to each of the three antennal segments indicate that the antennal lobe receives input from flagellar olfactory neurons exclusively, while the antennal mechanosensory and motor center is innervated by mechanosensory neurons from the whole antenna, comprising the flagellum, pedicle, and scape. The terminals in the mechanosensory and motor center are organized in segregated zones relating to the origin of neurons. The flagellar mechanosensory axons target anterior zones, while the pedicular and scapal axons terminate in posterior zones. In the ventral nerve cord, the processes from the antennal sensory neurons terminate in the motor area of the thoracic ganglia, suggesting a close connection with motor neurons. Taken together, the numerous neuropils innervated by axons both from the antenna and labial palp indicate the multiple roles these sensory organs serve in insect behavior.

Keywords: Mythimna separata, antenna, labial-palp pit organ, olfaction, mechanosensation, central projections, central nervous system 


\section{INTRODUCTION}

In moths, the antennae and the labial palps represent two prominent appendages for sensory input, involved in multiple behaviors, such as foraging, orientation, feeding, mating, and flight (Schneider, 1964; Guerenstein and Hildebrand, 2008; Krishnan and Sane, 2015). The labial-palp pit organ (LPO), located on the tip of each labial palp, possesses olfactory sensilla specialized for sensing carbon dioxide $\left(\mathrm{CO}_{2}\right.$, Guerenstein and Hildebrand, 2008). Previous findings, in both the pyralid moth, Cactoblastis cactorum, and sphingid moth, Manduca sexta, have shown that sensing of $\mathrm{CO}_{2}$ mediates oviposition and nectar feeding behaviors (Stange, 1997; Guerenstein et al., 2004). Axons of LPO sensory neurons project via the labial nerve to three main regions of the central nervous system: (1) to the LPO glomerulus (LPOG) in each antennal lobe, the primary olfactory center in the insect brain, (2) to the gnathal ganglion, and (3) to the ventral nerve cord (Bogner et al., 1986; Kent et al., 1986; Zhao et al., 2013). The antennal axons, on the other hand, target (1) all glomeruli of the ipsilateral antennal lobe except for the LPOG, (2) the antennal mechanosensory and motor center (AMMC), (3) the gnathal ganglion, and (4) the ventral nerve cord (Xie et al., 2016). Whereas the LPO houses sensory neurons tuned to one odor cue only, i.e., $\mathrm{CO}_{2}$, the antennal neurons are tuned not only to a large amount of odor stimuli but to several sensory modalities including taste, mechano-sensation, humidity, and temperature as well (Schneider, 1964; Altner and Loftus, 1985; Nishikawa et al., 1995; Jørgensen et al., 2006; Guerenstein and Hildebrand, 2008; Popescu et al., 2013; Enjin et al., 2016; Frank et al., 2017). The numerous odor neurons are housed inside various types of olfactory sensilla situated on the flagellum, such as trichoid sensilla, basiconic sensilla, and coeloconic sensilla (Schneider, 1964; Keil, 1999). These olfactory sensilla, being involved in detecting a variety of pheromones and host volatiles (de Bruyne et al., 2001; Christensen and Hildebrand, 2002), project their sensory axons exclusively into the antennal-lobe glomeruli. Furthermore, olfactory sensory neurons expressing a distinct odorant receptor type project to one or two glomeruli (Gao et al., 2000; Vosshall et al., 2000; reviewed by Christensen and Hildebrand, 2002).

In the moths Heliothis virescens and Spodoptera littoralis, antennal gustatory neurons, housed in sensilla chaetica, are reported to project into a subregion of the gnathal ganglion named the gustatory area of the moth brain (Jørgensen et al., 2006; Popescu et al., 2013). No thermo- or hygroreceptor neurons have so far been identified on the moth antenna. In the cockroach Periplaneta americana and the fruit fly Drosophila melanogaster, however, such neurons are encapsulated inside grooved hairs and pegs and project to glomeruli in the ventroposterior part of the antennal lobe (Nishikawa et al., 1995; Enjin et al., 2016). Antennal mechanosensory neurons are located in a variety of sensilla, including Böhm bristles, sensilla of Johnston's organ, and the above-mentioned s. chaetica, which are located on the flagellum. The mechanosensory neurons housed by s. chaetica, which are co-localized with chemosensory neurons, have large-diameter fibers targeting the AMMC (Jørgensen et al., 2006). Böhm bristles are located on the scape and pedicel, at the joint of scape-head and scape-pedicel, respectively. They detect the position of the antennae and control the insect steering during flight (Sane et al., 2007; Krishnan et al., 2012). Axons originating from Böhm bristles terminate in the AMMC (Krishnan et al., 2012). Johnston's organ, which is located at the inner surface of the pedicel, houses chordotonal neurons sensing a wide range of airflow vibrations or gravity (Yorozu et al., 2009; Dieudonné et al., 2014). Axons of Johnston's organ neurons (JONs) in the D. melanogaster, and honey bee, Apis mellifera, are found to be broadly distributed in several areas, including the AMMC (which is called the dorsal lobe in A. mellifera), dorsal part of the gnathal ganglion, and ventroposterior protocerebrum (Kamikouchi et al., 2006; Ai et al., 2007). Overall, antennal neurons housed inside morphologically specific sensillum categories detect distinct sensory modalities and project to different areas in the central nervous system, allowing the behaving insect to process the input from the different stimuli simultaneously.

The oriental armyworm moth, Mythimna separata (Walker) (Lepidoptera: Noctuidae) is a migratory, polyphagous pest, feeding on numerous plants in worldwide areas, including wheat, rice, corn, cotton, beans, as well as many vegetables (Lin, 1990; Jiang et al., 2014). Like other moth species, My. separata relies on olfaction for locating food and mates (He et al., 2017). In addition, My. separata is a migratory species, displacing seasonally over long distances (Chen et al., 1989; Feng et al., 2008; Zhao et al., 2009; Zhang et al., 2013). The multiple categories of antennal sensilla sensing different stimulus modalities simultaneously are probably an essential element for guiding this kind of behavior. However, the detailed projection pathways of the antennal sensory neurons in the central nervous system of My. separata are unknown.

Mythimna separata possesses a pair of typical filiform antennae, bearing numerous sensilla of different morphological types. On the flagellum, typical categories include s. chaetica, s. trichoidea, s. basiconica, and s. coeloconica (Chang et al., 2015). On the two basal segments, the scape and pedicel, there are many Böhm bristles (Chang et al., 2015). In addition to the antennal sensilla, Johnston's organ constitutes a particular structure housing numerous sensory neurons. Like other noctuid moths, My. separata possesses a large number of LPO sensilla as well (Dong et al., 2014). In the present study, the central projections from these sensory organs were investigated by using fluorescent staining combined with confocal microscopy and digital imaging reconstructions. The results provide a comprehensive map of the antennal and labial sensory neurons in the central nervous system of $M y$. separata and will ultimately aid understanding of the neuronal processes controlling multiple behaviors.

\section{MATERIALS AND METHODS}

\section{Insect Rearing}

Female and male adults of My. separata were used for the experiments. Larvae were reared on an artificial diet in the laboratory under the conditions of $27^{\circ} \mathrm{C}, 70 \%$ relative humidity, 
and a 16/8 h light/dark cycle. Adults were fed a $10 \%$ honey solution.

\section{Scanning Electron Microscopy}

An environmental scanning electron microscope was used to observe the antennal sensilla. The antennae were fixed in 2.5\% glutaraldehyde and then dehydrated with graded ethanol series (50, 70, 90, 96, and 100\%). After being mounted on a stub, the antennae were air-dried and gold-coated with sputter-coating before being examined with the electron microscope.

\section{Staining of Antennal Sensory Neurons}

The antennal sensory neurons were mapped by utilizing different staining experiments including (1) dye applied to the base of the scape, visualizing all sensory neurons, (2) dye applied to the base of the pedicel, visualizing all neurons except for those on the scape, (3) dye applied to the base of the flagellum, visualizing the flagellar neurons exclusively, (4) dye applied to cut s. trichoidea on the flagellum, visualizing a selection of olfactory afferents, (5) dye applied to cut sensilla forming the bristle of the pedicel, plus sensilla of the Johnston's organ, (6) dye applied to sensilla of the bristle on the scape. In all the staining experiments, the adult insect was fixed in a plastic tube with dental wax so that the head was exposed. In the first three experiments, the antenna was cut at the base of the scape, pedicel, and flagellum, respectively, as explained above. Crystals of the fluorescent dye, tetramethylrhodamine dextran (MicroRuby, Molecular Probes; Invitrogen, Eugene, OR, United States) were applied at the cut surface by using a needle. In the last three experiments mentioned, dye crystals were applied onto cut sensilla in the relevant regions. After all kinds of staining, the animal was placed in the fridge with a moist filter paper overnight allowing transportation of the dye in the sensory axons. On the second day, the brain and ventral nerve cord was dissected out in Ringer's saline, and then fixed for $1 \mathrm{~h}$ in $4 \%$ paraformaldehyde in $0.1 \mathrm{M}$ phosphate-buffered saline (PBS, pH 7.4). Following dehydration with an ascending ethanol series, the preparations were cleared in methylsalicylate, and mounted in Permount in perforated aluminum slides with two glass coverslips. In addition to the staining experiments described above, one experiment of retrograde neural labeling was performed. Here, dye was applied onto the cut end of the antennal nerve close to its entrance into the antennal lobe, in order to visualize the sensory neurons of the Johnston's organ.

\section{Staining of Sensory Neurons Projecting from the LPO}

The sensory neurons originating in the LPO were labeled by dye being applied onto the cut tip of the most peripheral segment of one labial palp. The following treatment of the stained preparations was similar to that described in the section above.

\section{Immunocytochemistry with Synapsin}

For visualizing central neuropil structures in the central nervous system of the moth, some of the mass-stained preparations were labeled by means of synapsin immunocytochemistry as well.
After being fixed in $4 \%$ paraformaldehyde in PBS and rinsed, as described above, the brain and ventral nerve cord was preincubated with 5\% NGS (Sigma, St. Louis, MO, United States) in $0.1 \mathrm{M}$ PBS containing $0.5 \%$ Triton X-100 (PBST; $0.1 \mathrm{M}$, $\mathrm{pH}$ 7.4) overnight at $4^{\circ} \mathrm{C}$. The primary antibody SYNORF1 (Developmental Studies Hybridoma Bank, University of Iowa), at a concentration of 1:100 (with 5\% NGS in PBST) was then applied, and the preparation was kept at $4^{\circ} \mathrm{C}$ for 5 days. Following rinse in PBS $6 \times 20 \mathrm{~min}$, the brain and ventral nerve cord was incubated in the secondary antibody, Cy2-conjugated anti-mouse (Invitrogen, Eugene, OR; dilution 1:300 with 1\% NGS in PBST), for 3 days at $4^{\circ} \mathrm{C}$. Finally, the preparation was washed $6 \times 20 \mathrm{~min}$ in PBS, dehydrated with graded ethanol series $(50,70,90,96$, each $10 \mathrm{~min}$, and $100 \% 2 \times 10 \mathrm{~min}$ ), cleared in methylsalicylate, and mounted in Permount, as described above.

\section{Image Acquisition and Analysis}

A confocal laser scanning microscope (LSM 780, META Zeiss, Jena, Germany) was used to obtain images of the sensory neurons and the neuropils. Two objectives were utilized, a Plan-Neofluar $10 \times / 0.3$ for large-scale images and a $20 \times / 0.51$ for higher resolution. The $\mathrm{Cy} 2$, displaying brain structures, was excited by an argon laser of 488-nm line and the Micro-Ruby, displaying the stained neurons, by a HeNe laser 543-nm line. The resolution of the confocal images was obtained at $1024 \times 1024$ voxels with intervals of 3 or $4 \mu \mathrm{m}$.

Identified neuropils within the brain and ventral nerve cord were reconstructed by using the Amira software (Amira 5.3, Visage Imaging, Fürth, Germany). The stained axons and neuronal processes were reconstructed manually as described previously by means of the skeleton tool of Amira (Zhao et al., 2016).

\section{RESULTS}

\section{Morphological Features of Sensilla and Neurons Confined to the Antenna}

The images from electron microscopy demonstrated that the antennae of $M y$. separata are covered by morphologically different categories of sensilla (Figure 1; Chang et al., 2015). The flagellum is the site for several types of sensilla including s. trichoidea, s. basiconica, s. coeloconica, and s. styloconica. The most abundant category is the s. trichoidea appearing as long hairs (Figures 1A,B) On the pedicel and scape, the Böhm bristles are gathered in clusters at the joints of the pedicel-scape and the scape-head, respectively (Figures 1A,C,E). There are two clusters of Böhm bristles on the pedicel and two on the scape, positioned opposite to each other (Figures 1C,E). The number of bristles on the scape is relatively large. Böhm bristles comprise short s. chaetica (indicated by arrows in Figures 1D,F) surrounded by many short seta (indicated by arrowheads in Figures 1D,F).

Among 12 attempted trials including dye applied to the proximal antennal nerve (AN), for labeling the peripheral part of the antennal sensory neuron, eight were successful. The retrograde staining revealed that the $\mathrm{AN}$ is organized into two sub-bundles formed mainly by axons of flagellar sensory 

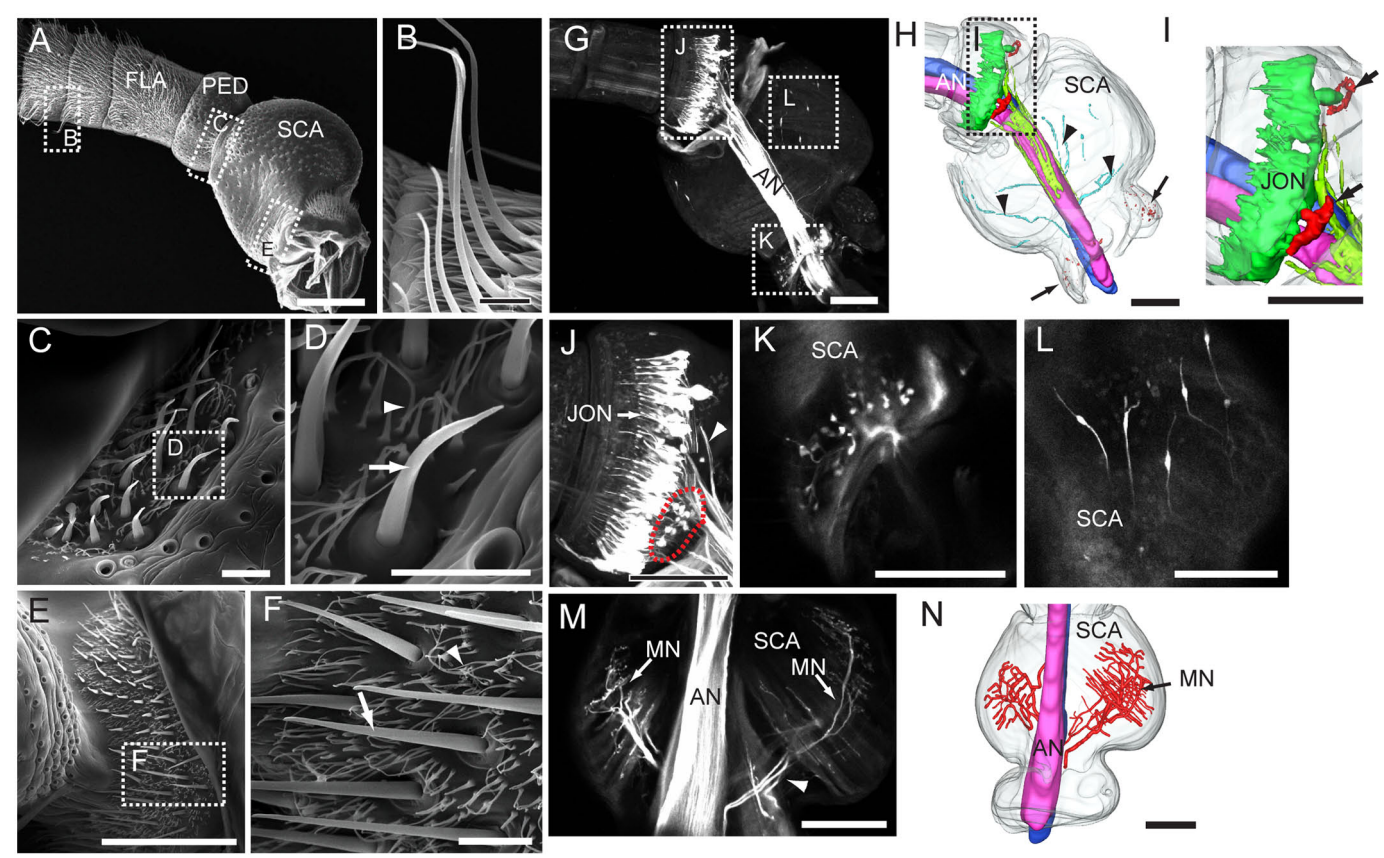

$\mathrm{N}$

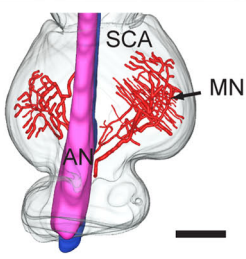

FIGURE 1 | Morphological properties of the antenna, including its segments, sensilla, and sensory neurons. (A) Proximal part of the antenna with all three segments: scape (SCA), pedicel (PED), and four annuli of the flagellum (FLA). (B) Sensilla trichoidea on the flagellum. (C,D) Böhm bristles on the pedicel. (E,F) Böhm bristles on the scape. (G) Confocal image of the antennal nerve (AN) and antennal sensory neurons of the pedicel and scape, visualized by retrograde staining.

(H) Three-dimensional reconstruction showing the stained AN and antennal sensory neurons of the pedicel and scape. Arrows indicate the sensory neurons of Böhm bristles on the scape. Arrowheads indicate axons of sensory neurons on the inner cuticular surface of the scape. (I) Three-dimensional reconstruction showing neurons of Johnston's organ (JONs) and sensory neurons of Böhm bristles on the pedicel (indicated by arrows). (J) Confocal image of JONs and sensory neurons of Böhm bristles on the pedicel (indicated by red dotted circle). Arrowhead indicates axons of JONs. (K) Confocal image of sensory neurons of Böhm bristles on the scape. (L) Confocal image of sensory neurons on the inner cuticular surface of the cuticle. (M) Confocal image of motor neurons (MN) linked to intrinsic muscles of the scape. (N) Three-dimensional reconstruction showing the motor neurons. Scale bars, (A,G-N) $100 \mu \mathrm{m}$. (B-F) $10 \mu \mathrm{m}$.

neurons (Figures 1G,H). At the proximal part of the pedicel, there is a large number of bipolar neurons confined to the Johnston's organ (Figures $\mathbf{1 G}, \mathbf{H}, \mathbf{J})$. The dendrites of these sensory neurons extend into the distal part of pedicel (Figure 1J) whereas their axons join the AN from a surrounding position (Figures 1H,J). The Böhm bristles, which were found both on the scape and pedicel, encapsulate sensory neurons extending their dendrites under the cuticular protrusion (scape: Figures 1G,K; pedicel: Figures 1G,J, indicated by red dotted circle line). Clusters of these sensory neurons are located opposite to each other, two on the scape and two on the pedicel (indicated by arrows in Figures $\mathbf{1 H}, \mathbf{I})$. In addition to sensory neurons of Böhm bristles, a few bipolar neurons were observed in the proximal part of the scape (Figures 1G,H,L). These neurons resemble those of the Johnston's organ by extending their dendrites toward the cuticle and having axons joining the AN (Figure $1 \mathrm{H}$ indicated by arrowheads). The AN also includes some motor neurons which innervate intrinsic muscles of the scape (Figures 1M,N).

\section{Overview of the Antennal-Axon Terminals in the Central Nervous System}

The gross innervation pattern of antennal sensory neurons in the central nervous system of My. separata was mapped from confocal images of 10 successfully stained preparations (among 21 trials), in which dye was applied to the base of the scape. The axons of all antennal afferents project via the AN to the ipsilateral side of the central nervous system, including the antennal lobe $(\mathrm{AL})$, lateral protocerebrum, AMMC, gnathal ganglion, plus the prothoracic and mesothoracic ganglion of the ventral nerve cord (Figures 2A,C,D and Table 1). Two kinds of additional staining experiments including dye applied to the base of the pedicel (20 trials) and flagellum (24 trials) resulted in 12 and 14 successfully stained preparations, respectively. All these preparations showed labeled projections in the regions mentioned above, including the $\mathrm{AL}$, lateral protocerebrum, AMMC, gnathal ganglion, and ventral nerve cord. However, whereas the specific innervation patterns in the AL and the lateral protocerebrum were similar across preparations stained from the three different antennal segments, they differed in the remaining target regions (Table 1). Males and females displayed identical projection patterns.

\section{Central Projection Patterns of Antennal Sensory Neurons in the AL and the Protocerebrum}

The most prominent target area of the antennal afferents was the AL (Figures 2, 3A-C). Here, in the primary olfactory center, the 

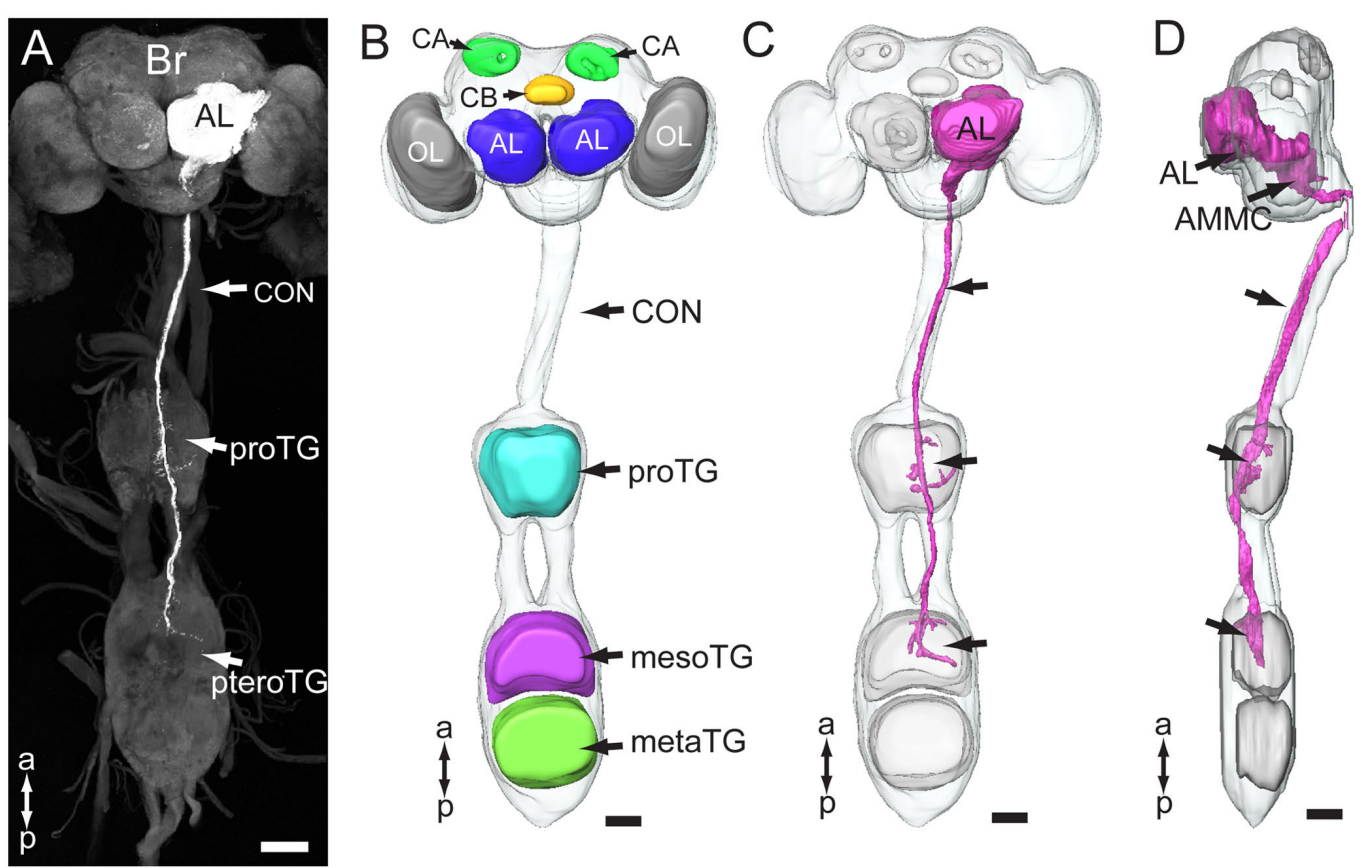

FIGURE 2 | Gross projection pathway of the antennal sensory neurons in the central nervous system of My. separata. (A) Confocal image of antennal axons terminating in the brain and ventral nerve cord. (B) Three-dimensional reconstruction showing prominent structures of the central nervous system. The brain and thoracic ganglia are linked by the long connective (CON). Brain neuropils include the optic lobes (OL), antennal lobes (AL), central body (CB), and calyx (CA). Ventral-cord neuropils include the thoracic ganglia, prothoracic ganglion (proTG), mesothoracic ganglion (mesoTG), and metathoracic ganglion (metaTG). The mesoTG and metaTG are fused forming the pterothoracic ganglion (pteroTG). (C,D) Three-dimensional reconstruction showing the overall projection pathway of the antennal sensory neurons in the central nervous system (frontal and lateral view, respectively). AMMC, antennal mechanosensory and motor center; Br, brain. a, anterior; p, posterior. Scale bars, $100 \mu \mathrm{m}$.

sensory axons form a characteristic pattern consisting of hollow spheres, established by terminals innervating the periphery of the AL glomeruli (Figures $\mathbf{3 A}-\mathbf{C}$ ). All glomeruli receive input from the antennal axons, except for one, which is located most ventrally in the AL (indicated by asterisk in Figure $3 \mathbf{B}$ ). The projection pattern in the $\mathrm{AL}$ was identical in all successfully stained preparations no matter if dye had been applied to the scape, pedicel, or flagellum (Table 1). Specific staining of selected s. trichoidea, situated on the flagellum, showed labeled axons innervating the AL glomeruli exclusively (Figure 3D and Table 1).

In addition to the numerous axons targeting the AL, a few stained fibers projected to the ipsilateral protocerebrum of the brain (Figures 3E,F,H,I and Table 1). This region, which is positioned laterally to the central body and anteriorly of the mushroom body calyx, was innervated by labeled processes projecting via the posterior $\mathrm{AL}$ (Figure $\mathbf{3 H}$ indicated by an arrowhead). Again, the projection pattern appeared similar in all stained preparations.

In addition to the terminals of sensory neurons, a big soma located at the posterior border of brain, on the ventrolateral side of esophagus, was stained (Figures $3 \mathbf{E}, \mathbf{G}$ ). Its primary neurite bifurcated; one branch targeted the AMMC and the other projected ventrally of the esophagus to the contralateral side (Figure 3I, indicated by an arrowhead). It may be a centrifugal neuron innervating sensilla located on the flagellum. Unfortunately, the terminals of these prominent neural processes could not be traced owing to the weak staining.

\section{Projection Pattern of Antennal Sensory Neurons in the AMMC}

A substantial bundle of the antennal axons bypasses the AL on its lateral side and targets the AMMC (Figure 4 and Table 1). According to the pattern of axon terminals, at least eight zones were identified, of which Zones 3, 4, and 8 partly overlap with the gnathal ganglion (Figures $4 \mathrm{~A}-\mathbf{F}$ ). Zone 1 is located ventro-posteriorly of the AL, and Zone 2 ventrally to Zone 1 (Figures 4A,D). Zone 3, which is located laterally of the tritocerebrum, close to the dorsal midline of the gnathal ganglion, receives projections of antennal axons bypassing Zone 1 (Figures 4A,D). Zone 4 is located medio-posteriorly to the ventral part of Zone 2 and receives projections of axons bypassing Zone 1 (Figures 4B,D). Zone 5 is located posteriorly of Zone 2 (Figures 4B,E), Zone 6 medio-posteriorly of the dorsal AMMC, and Zone 7 medio-posteriorly of the ventral AMMC (Figures 4C,F). Zone 8, which borders with the midline of the gnathal ganglion and receives projections bypassing Zone 7 , is located ventro-medially of Zones 4 and 7 (Figures 4C,D).

The preparations stained at the base of the scape and the pedicel showed similar projections patterns including labeled axons terminating in all eight zones of the AMMC 


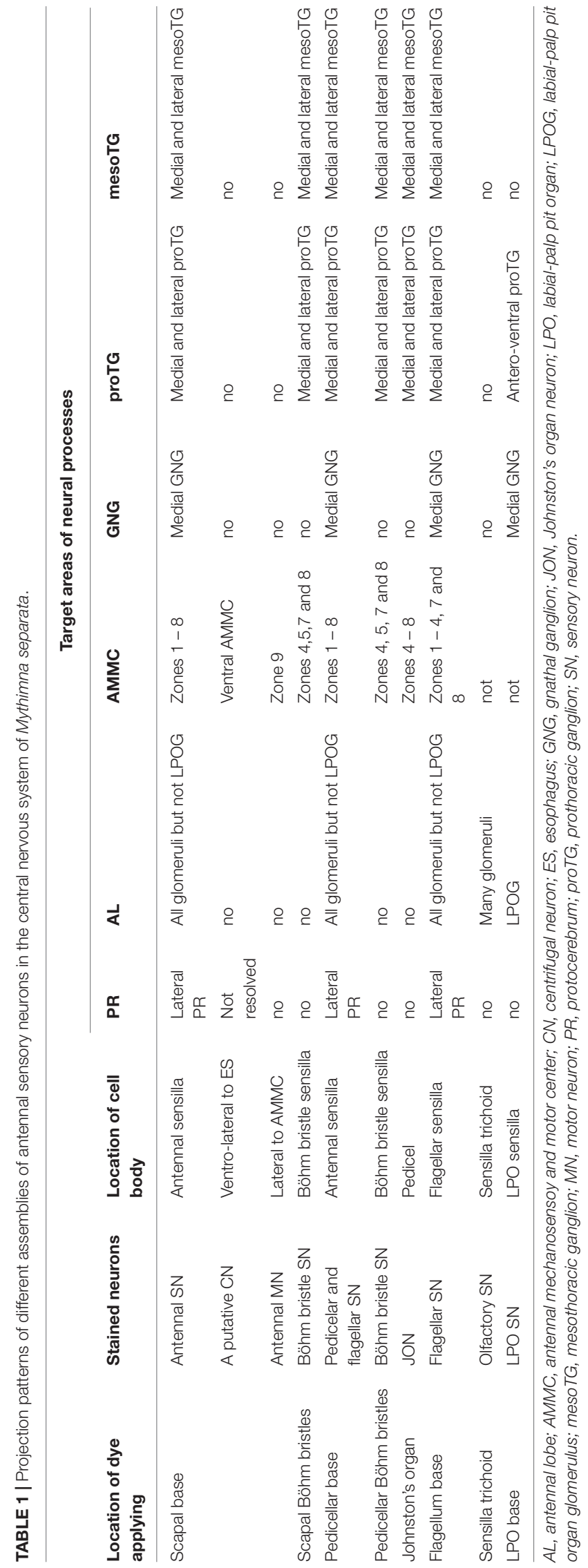

(Figures 4A1-F1,A2-F2). The preparations stained at the base of the flagellum, on the other hand, showed labeled axons terminating in only six zones, i.e., Zones $1-4$, plus 7 and 8 (Figures 4A3-F3).

Specific staining of Böhm bristles demonstrated that their sensory axons innervate almost the same areas, i.e., Zones 4, 5 , 7 , and 8 , whereas specific staining of sensilla on the Johnstons' organ gave rise to labeled terminals in Zones $4-8$ (Figures 4G-K and Table 1). The preparations stained from the base of the scape showed a few labeled somata as well (Figure 4A1). According to previous reports from Ma. sexta, these are probably motor neurons (Kloppenburg et al., 1997). As demonstrated in Figures 4D1-F1, these neurons arborize mainly in a region located ventro-posteriorly in the AMMC (Zone 9). Notably, none of these motor neurons were stained when dye was applied at the base of the pedicel and the flagellum.

\section{Projection Pattern of Antennal Neurons in the Thoracic Ganglia}

Within the axon bundle bypassing the AL, there are some long fibers forming two descending tracts that project into the ventral nerve cord. These paths, named T1 and T2, are separated when they bypass Zone 4 and 8 , but fuse before entering the ventral nerve cord (Figures 4D-F).

In the ventral nerve cord, the antennal axons project via the ipsilateral connective, first to the prothoracic ganglion (proTG) and then to the mesothoracic ganglion (mesoTG, Figure 5 and Table 1). In the proTG, the axons pass near the midline and give off processes in the central area of the neuropil (Figure 5A). A few processes cross the midline to the contralateral side and some project to the lateral part of the neuropil (Figure 5B, indicated by arrows). The axons project further to the central area of the mesoTG (Figure 5C). Here, the axons make a loop and at the same time give off processes. As in the proTG, a few processes cross the midline to the contralateral side and some project to the lateral part of the neuropil (Figure 5D, indicated by arrows).

The preparations stained at the base of the scape and the pedicel showed similar projection patterns in the ventral nerve cord (Figures 5A1-D1,A2-D2), whereas those stained at the base of flagellum showed labeling of some axons of the T1 tract only. This fiber bundle, which is substantially thinner than the whole T1 tract, can be observed as it exits the gnathal ganglion and projects to the ventral nerve cord (Figures 4D3-F3, 5A3-D3).

\section{Central Projections of the LPO Sensory Neurons}

Among 33 preparations attempted labeled by applying dye into the LPO, 16 were successful. The stained axons of the LPO sensory neurons projected via the ipsilateral labial palp nerve and terminated in three main areas of the central nervous system: (1) LPOG in each AL, (2) the gnathal ganglion, and (3) the proTG of the ventral nerve cord (Figure 6). The most prominent target region was the LPOG. After entering the gnathal ganglion, a substantial portion of the stained axons divided into two bundles, the antenno-gnathal tracts, each projecting to the LPOG in one $\mathrm{AL}$ (Figures 6A,E,F). Here, the stained terminals filled the whole 

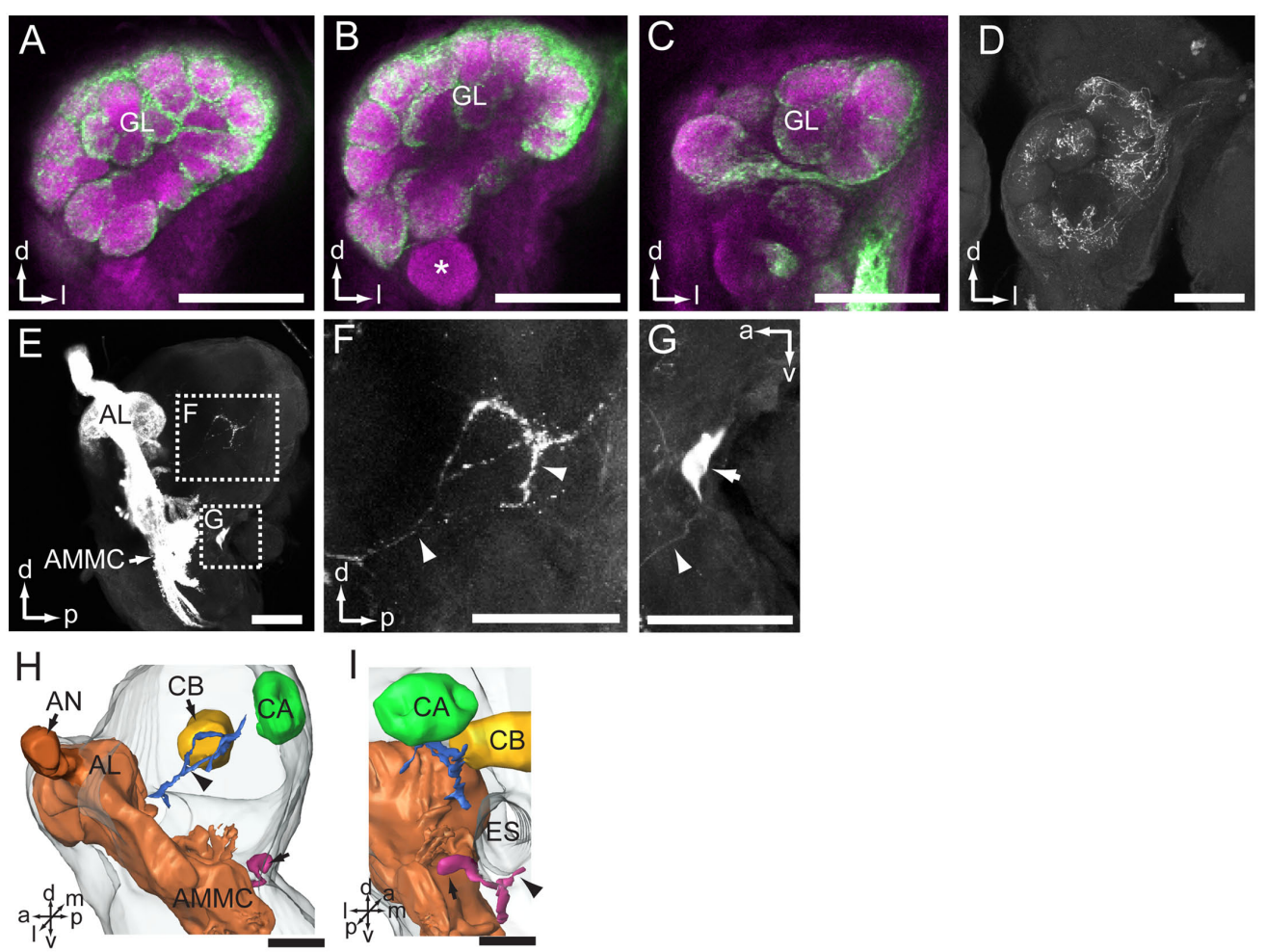

FIGURE 3 | Central projections of antennal sensory neurons in the antennal lobe (AL) and lateral protocerebrum of the brain. (A-C) Confocal images of antennal sensory neurons (green) targeting the core of AL glomeruli (GL, magenta). Asterisk in (B) indicates one large GL devoid of antennal axons. (D) Projections of sensory neurons located on the flagellum, visualized via selective staining of sensilla trichoidea. (E) Sagittal view of antennal sensory projections in the brain, targeting the AL, $\mathrm{AMMC}$, and the lateral protocerebrum. (F) Enlarged image of the stained axons in the lateral protocerebrum. Arrowheads indicate neuron processes. (G) One big soma located posteriorly in the brain, under the esophagus (ES), was stained (arrow). The arrowhead indicates the neurite. (H,I) Three-dimensional reconstruction showing projections of antennal sensory neurons in the brain (lateral and posterior view, respectively). The arrow indicates the cell body. The arrowhead indicates the processes. (I) AMMC, antennal mechanosensory and motor center; AN, antennal nerve; CA, calyx; CB, central body. a, anterior; d, dorsal; I, lateral; m, medial; p, posterior; v, ventral. Scale bars, $100 \mu \mathrm{m}$.

LPOG, and a few processes even extended outside the glomerulus (Figures 6C,D, indicated by arrows). Staining of antennal sensory neurons demonstrated that the LPOG does not receive any input from this neuronal category (Figure 3B, indicated by an asterisk). The second target region of LPO axons, the gnathal ganglion, is innervated by processes terminating mainly in the ipsilateral neuropil. Only a few stained branches extended to the contralateral side (Figures 6B,E, arrowheads). The third target region, the proTG of the ventral nerve cord, was innervated by axons leaving the gnathal ganglion via the ipsilateral connective (Figures 6G-I). In the proTG, the stained axons terminated in the anterior part of the neuropil forming a loop (Figure 6G). Males and females showed identical staining patterns in all three regions of the central nervous system.

\section{DISCUSSION}

\section{Antennal Sensory Neurons}

Data obtained by electron microscopy showed that a large number of sensilla, including different morphological categories, are present on the flagellum of My. separata, whereas the pedicel and scape carry mainly Böhm bristles. These data are in full agreement with previous reports on the same species (Chang et al., 2015) and other insects (Schneider, 1964; Keil, 1999). The retrograde staining experiments visualized the antennal sensory neurons housed by the sensilla and their axons forming the AN. In addition, a large number of JONs located close to the inner surface of the pedicel, plus a few bipolar neurons at the inner surface of the scape were observed. Since no obvious sensilla were found on the external surface of the scape, these bipolar neurons may act as proprioceptors for the scape. Their specific function remains to be explored, however. Interestingly, the Böhm bristles and JONs are similar to those of Ma. sexta, which are found to work as mechanosensory neurons detecting movement and position of the antenna (Sane et al., 2007; Krishnan et al., 2012; Dieudonné et al., 2014). Thus, they are probably involved in flight control (Sane et al., 2007). The antennae of the monarch butterfly possess magnetic sensors and a circadian clock facilitating optimal orientation during migration (Merlin et al., 2009; Guerra et al., 2014). My. separata might actually possess corresponding arrangements as well, since it is reported to maintain consistent directions when migrating (Xu et al., 2017). Generally, the antennal sensilla of $M y$. separata comprise several morphological 

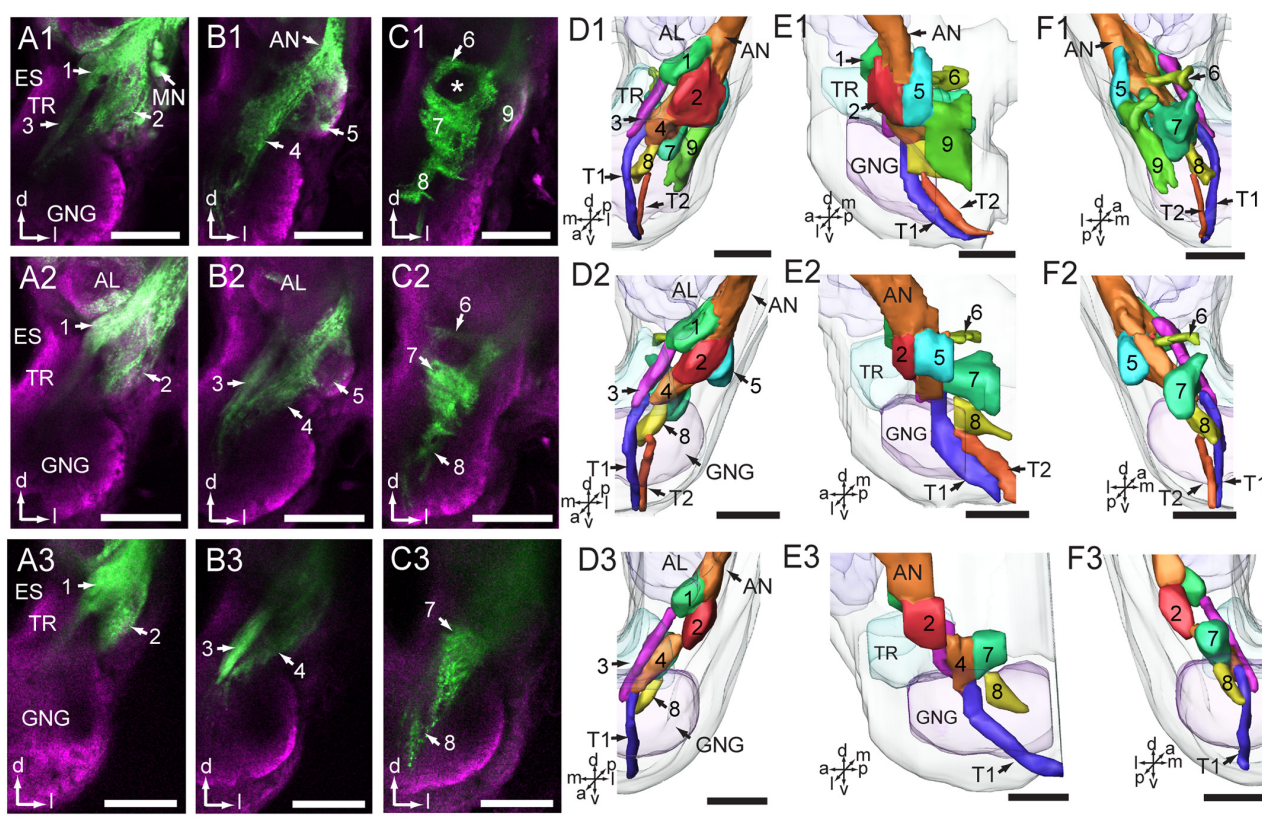

F2
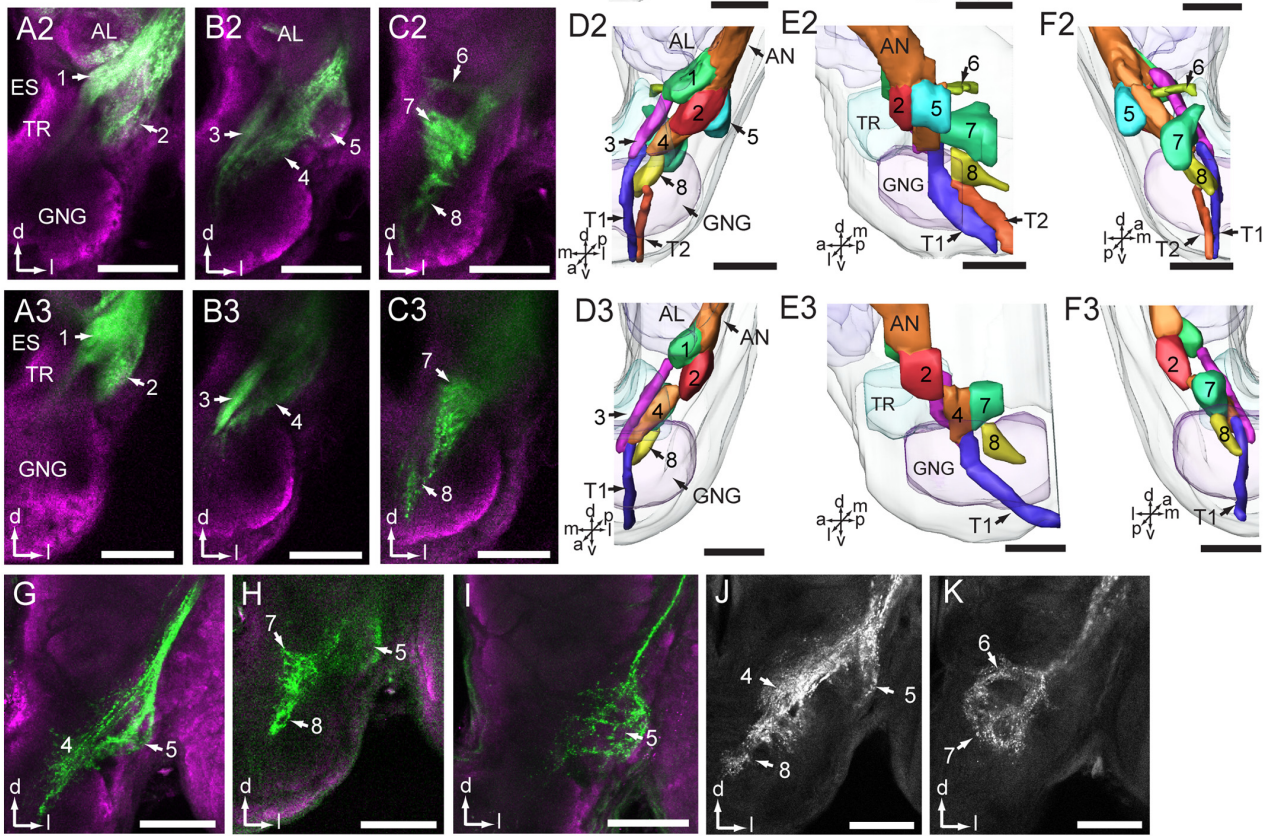

FIGURE 4 | Projections of antennal sensory neurons in the antennal mechanosensory and motor center (AMMC). (A1-C1) Confocal images of antennal sensory projections in the AMMC, stained from the base of the scape. (D1-F1) Three-dimensional reconstructions of the sensory projections shown in (A1-C1). Frontal view (D1), lateral view (E1), and posterior view (F1). (A2-C2) Confocal images of antennal sensory projections in the AMMC, stained from the base of the pedicel. (D2-F2) Three-dimensional reconstruction of the sensory projections shown in (A2-C2). Frontal view (D2), lateral view (E2), and posterior view (F2). (A3-C3) Confocal images of antennal sensory projections in the AMMC, stained from the base of the flagellum. (D3-F3) Three-dimensional reconstruction of the sensory projections shown in (A3-C3). Frontal view (D3), lateral view (E3), and posterior view (F3). $\mathbf{( G , H ) ~ N e u r o n ~ p r o j e c t i o n s ~ o f ~ s e l e c t i v e l y ~ s t a i n e d ~ B o ̈ h m ~ b r i s t l e s ~ l o c a t e d ~ o n ~}$ the scape. (I) Neuron projections of selectively stained Böhm bristles located on the pedicel. (J,K) Central projections of Johnston's organ neurons. The numbers 1-8 in (D,E, F) indicate zones of the AMMC innervated by sub-groups of sensory axons. Number 9 indicates an area innervated by motor neurons. T1 and T2 indicate the descending tracts of antennal sensory neurons projecting to the ventral nerve cord. AL, antennal lobe; AN, antennal nerve; ES, esophagus; GNG, gnathal ganglion; MN, motor neuron; TR, tritocerebrum. a, anterior; d, dorsal; I, lateral; m, medial; p, posterior; v, ventral. Scale bars, $100 \mu \mathrm{m}$.

types designed to detect different modalities, which in turn allow My. separata to perceive simultaneous input about the complex environment.

\section{Projection Pattern of the Antennal Sensory Neurons in the Central Nervous System}

By combining electron microscopy with different mass staining experiments, including dye applied to the base of the scape, pedicel, and flagellum, respectively, we obtained a general overview of all antennal projections in the central nervous system as well as more detailed maps visualizing the target regions of sensory axons housed inside sensillum types located on the different antennal segments. The general staining pattern of all antennal afferents covered innervations in several ipsilateral regions of the central nervous system including the $\mathrm{AL}$, protocerebrum, AMMC, gnathal ganglion, proTG, and mesoTG.
Such a widespread projection pattern of the antennal axons was also observed in the locust, Locusta migratoria, blowfly, Calliphora erythrocephala, blood-sucking bug, Rhodnius prolixus, and mirid bug, Apolygus lucorum (Bräunig et al., 1983; Nässel et al., 1984; Barrozo et al., 2009; Xie et al., 2016). The multiple targeting areas may correspond to the different sensory modalities being detected by the antennal sensory neurons. Such a multisensory organ as the antennae of $M y$. separata provides the flying insect with detailed information about the external world.

In addition to the afferent axons, a putative centrifugal neuron innervating the antenna was stained as well. This kind of neuron has not been reported in any insect species so far.

\section{The $\mathrm{AL}$ and the Protocerebrum}

Among all innervated regions, the AL was the most heavily stained area. This indicates a key role of the antenna in olfaction. The innervating pattern in the $\mathrm{AL}$ was similar 

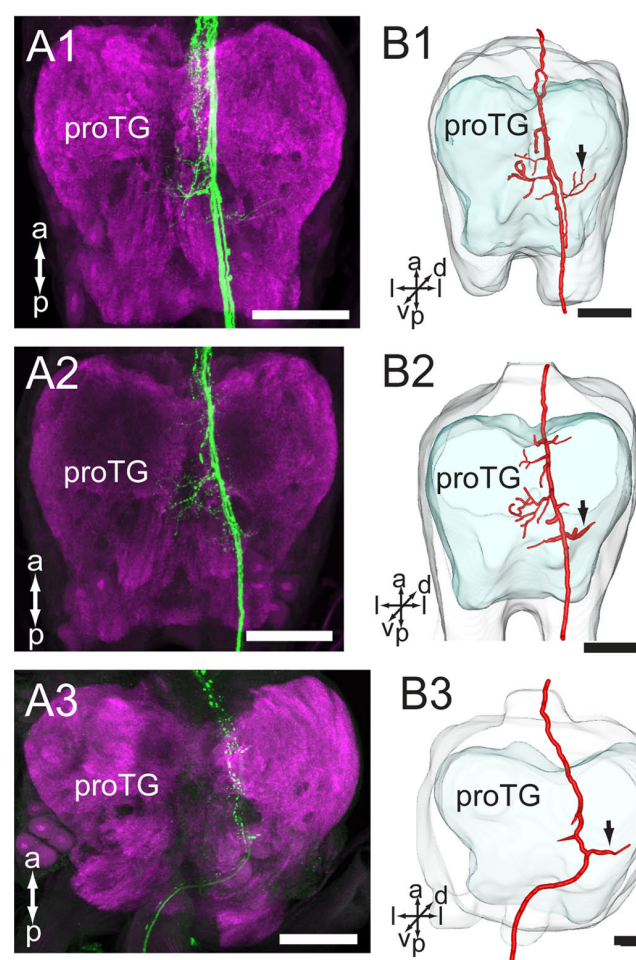
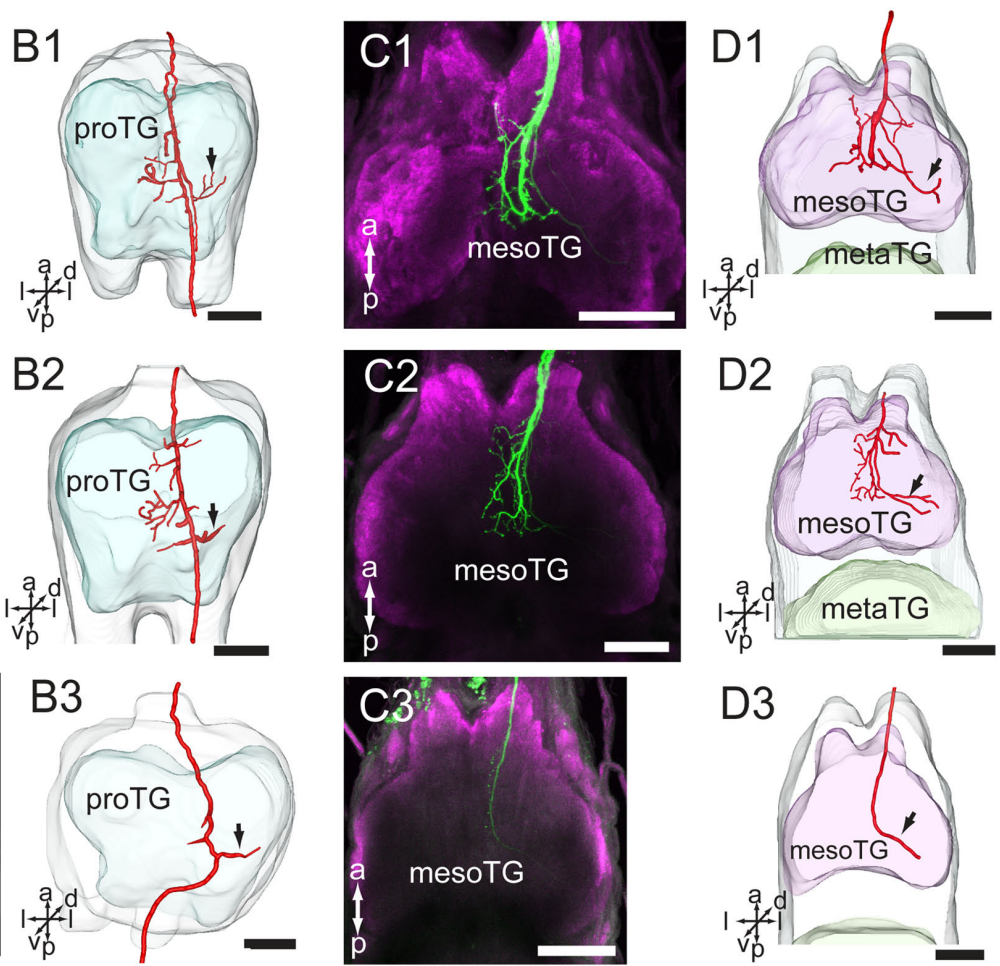
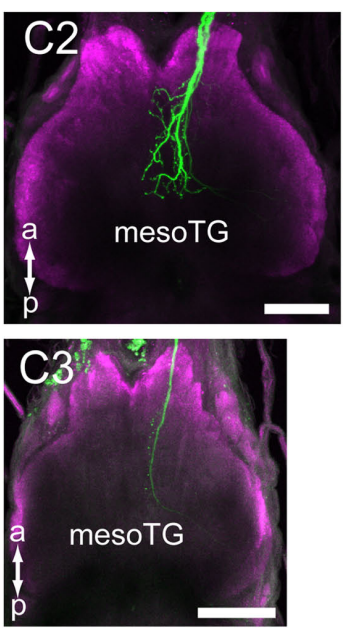
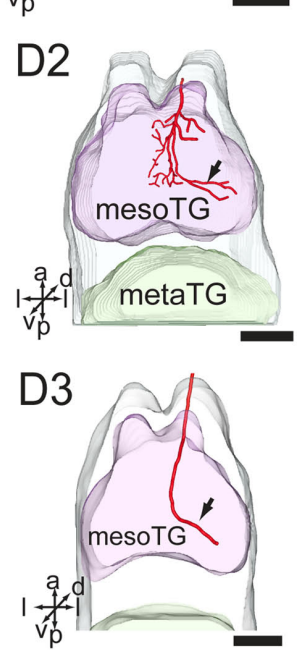

FIGURE 5 | Projections of the antennal sensory neurons in the thoracic ganglia (TG) of the ventral nerve cord. (A) Confocal images of axon projections (green) in the proTG (magenta). (B) Three-dimensional reconstructions of the sensory axons shown in (A). (C) Confocal images of axon projections (green) in the mesoTG (magenta). (D) Three-dimensional reconstruction of the sensory axons shown in (C). In (A1-A3), dye was applied at the base of the scape, pedicel and flagellum, respectively. In (C1-C3), dye was applied at the base of the scape, pedicel and flagellum, respectively. Arrow indicates the long processes extending to the lateral part of the ganglion. mesoTG, mesothoracic ganglion; proTG: prothoracic ganglion. a, anterior; d, dorsal; I, lateral; p, posterior; v, ventral. Scale bars, 100 $\mu$ m.

in all three types of anterograde mass staining experiments, suggesting that the AL receives input from flagellar sensory neurons. In addition, specific staining of selected s. trichoidea showed labeling in AL glomeruli only, indicating that sensory neurons housed inside this numerous population of antennal sensilla terminate in the AL glomeruli exclusively. Such a projection pattern of olfactory sensory neurons has been found in many other insect species within a wide range of orders, for instance, dragonfly Libellula depressa (Rebora et al., 2013), cricket Gryllus bimaculatus (Yoritsune and Aonuma, 2012), cockroach Periplaneta americana (Nishino et al., 2005), louse Columbicola columbae (Crespo and Vickers, 2012), bugs Euschistus heros, R. prolixus, and Apolygus lucorum (Kristoffersen et al., 2008; Barrozo et al., 2009; Xie et al., 2016), aphid Sitobion avenae, Metopolophium dirhodum, and Acyrthosiphon pisum (Kristoffersen et al., 2008; Kollmann et al., 2011), psyllid Trioza apicalis (Kristoffersen et al., 2008), beetle Tribolium castaneum (Dippel et al., 2016), A. mellifera (Nishino et al., 2009), moth Helicoverpa armigera (Zhao et al., 2016), D. melanogaster (Stocker et al., 1983), Aedes aegypti, and Anopheles gambiae (Distler and Boeckh, 1997; Anton et al., 2003; Ignell et al., 2005).

A few stained axons projected to a region in the lateral protocerebrum. Similarly to the axons targeting the AL, they seem to originate from the flagellum. Projections of antennal sensory neurons targeting the protocerebrum were also observed in other insects, for instance, L. depressa, Apolygus lucorum, C. erythrocephala, D. melanogaste, A. mellifera, Ae. aegypti, and An. gambiae (Nässel et al., 1984; Ignell et al., 2005; Kamikouchi et al., 2006; Ai et al., 2007; Nishino et al., 2009; Rebora et al., 2013; Xie et al., 2016). However, these are mechanosensory neurons originating from the pedicel: in C. erythrocephala from the campaniform sensilla and in D. menlanogaster and A. mellifera from the Johnston's organ (Nässel et al., 1984; Kamikouchi et al., 2006; Ai et al., 2007). In My. separata, on the other hand, the type of sensilla housing these neurons has not yet been identified.

\section{The AMMC}

The AMMC was the second most heavily stained area in the brain of My. separata. As its name suggests, the AMMC is the center for input from antennal mechanosensory neurons and for controlling movement of the antenna. Similarly to the general arrangement in the AL, the projection pattern of antennal sensory neurons in the AMMC is to a large extent conserved across a wide range of insects (Ignell et al., 2005; Nishino et al., 2005; Kamikouchi et al., 2006; Kristoffersen et al., 2008; Barrozo et al., 2009; Nishino et al., 2009; Kollmann et al., 2011; Crespo and Vickers, 2012; Yoritsune and Aonuma, 2012; Rebora et al., 2013; Xie et al., 2016; Zhao et al., 2016). 

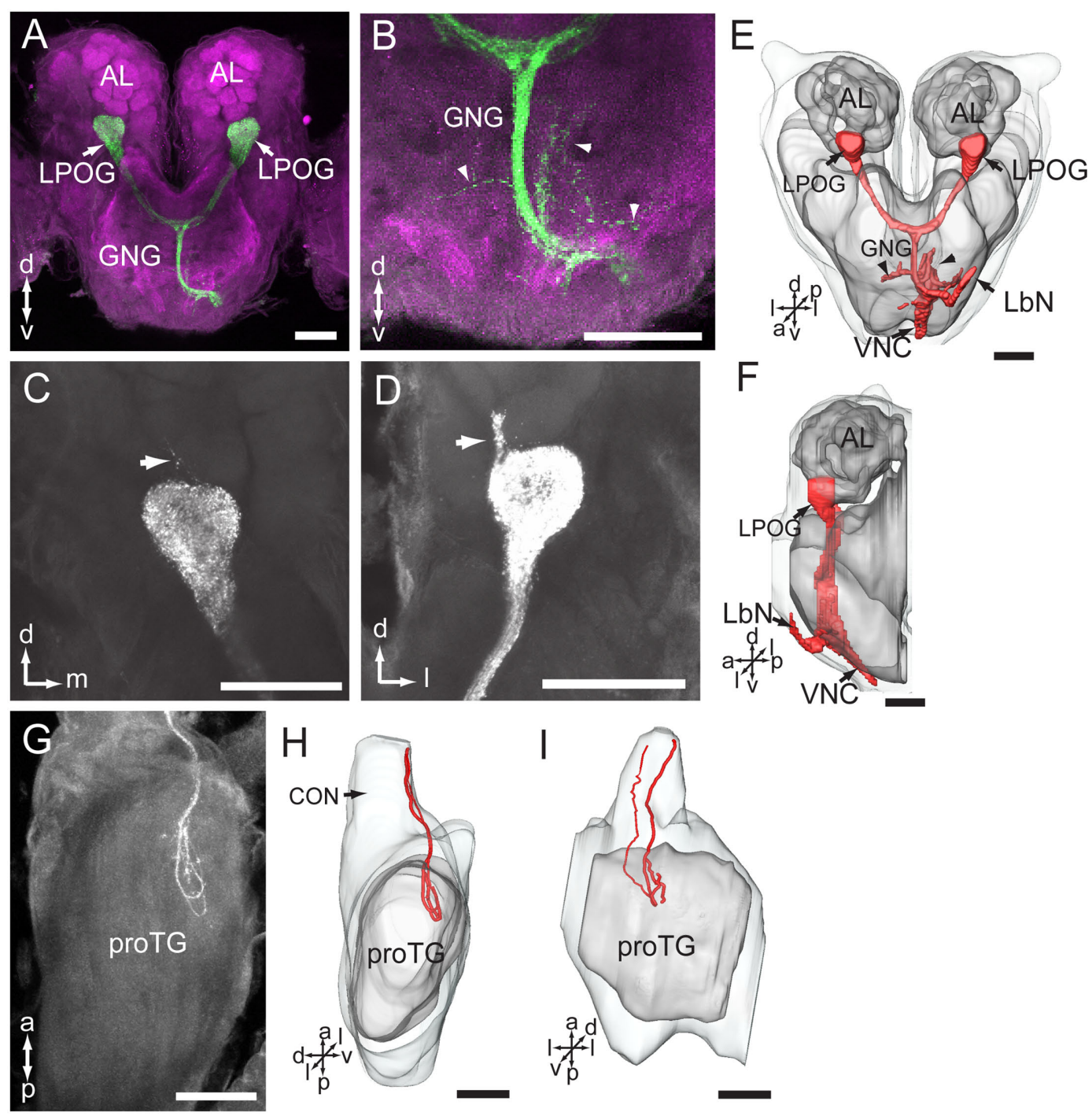

FIGURE 6 | Central pathway of sensory neurons located in the labial palp organ (LPO). (A) Confocal image showing the main target region of the LPO sensory neurons (green), i.e., the LPO glomerulus (LPOG) in each antennal lobe (AL; magenta). (B) Enlarged image showing the sensory processes in the gnathal ganglion (GNG; arrowheads). (C,D) Confocal images showing terminals of stained axons innervating the entire LPOG, with a few processes outside of LPOG (arrows). (E,F) Three-dimensional reconstructions of the sensory pathway shown in (A,B). (E) In frontal view, (F) in lateral view. (G) Confocal image showing axon projections in the prothoracic ganglion (TG). (H,I) Three-dimensional reconstructions of the axon terminals shown in (G). (H) In lateral view, (I) in ventral view. CON, connective; LbN, labial nerve; VNC, ventral nerve cord. a, anterior; d, dorsal; I, lateral; p, posterior; v, ventral. Scale bars, $100 \mu \mathrm{m}$.

Altogether, the antennal sensory axons showed terminal projection into eight zones of AMMC of My. separata. However, the more detailed staining patterns in the AMMC differed when dye was applied to the base of the flagellum versus scape and pedicel. The fact that flagellar axons innervated Zones $1-4$, plus 7 and 8, axons of Böhm bristles, located on the pedicel and scape, targeted Zones 4, 5, 7, and 8, whereas axons of JON terminated in Zones $4-8$, suggests that distinct zones receive different signal categories.

Previous studies on the moths Heliothis virescens and S. littoralis including specific staining of s. chaetica have shown that their sensory neurons project to the AMMC and gnathal ganglion (Jørgensen et al., 2006; Popescu et al., 2013). The s. chaetica, situated on the flagellum, are contact chemosensors of moths, each housing four gustatory and one mechanosensory neuron (Jørgensen et al., 2006; Popescu et al., 2013). The different neurons project to distinct areas (Popescu et al., 2013). Their target areas in the AMMC seem to correspond to Zones 1 - 3 of My. separata.

The finding that sensory neurons of Böhm bristles, situated on the pedicel and scape, project to the same areas corresponds to previous reports from Ma. sexta. The previous studies showed that these neurons are involved in control of flight (Sane et al., 2007; Krishnan et al., 2012; Dieudonné et al., 2014). Concerning the JONs, it has been found, from investigations on D. melanogaster and A. mellifera, that these neurons also project 
to spatially segregated zones, and that the projection pattern is related to the locations of cell bodies (Kamikouchi et al., 2006; Ai et al., 2007). In D. melanogaster, it has been demonstrated that two distinct zones (named A and B) of the AMMC are responsible for near-field sound, and two others (named $\mathrm{C}$ and $\mathrm{E}$ ) for gravitational forces and wind-induced deflections (Kamikouchi et al., 2009; Yorozu et al., 2009).

\section{The Thoracic Ganglia}

The fact that the projection pattern in the two ganglia was similar when staining from the scape and pedicel, and slightly reduced when staining from the flagellum, indicates that the labeled axons in the ventral nerve cord originate from the flagellum and pedicel exclusively. The general projection pattern of antennal afferents terminating in the proTG and mesoTG is similar to that observed in other insect species (Bräunig et al., 1983; Nässel et al., 1984; Barrozo et al., 2009; Xie et al., 2016). In particular, some of the sensory neurons originating from the flagellum and pedicel of $M y$. separata extend a few long processes to lateral parts of the neuropils, known to be motor centers of the thorax (Nässel et al., 1984). This particular pattern is comparable with sensory neurons originating from campaniform sensilla on the pedicel in C. erythrocephala (Nässel et al., 1984). Interestingly, the axons of these neurons give off long bilateral branches into regions of the thoracic ganglia that act as leg motor centers (Nässel et al., 1984). Such an arrangement of antennal sensory neurons indicates a relatively close connection between the periphery and central motor areas presumably facilitating control of locomotion. The sensillum type housing the long antennal projections of $M y$. separata has not yet been identified. The flagellar sensory neurons previously described in Heliothis virescens and S. littoralis were housed by s. chaetica (Jørgensen et al., 2006; Popescu et al., 2013). Since none of these neurons sent projections to the ventral nerve cord, it seems as if the descending sensory neurons of the flagellum originate from other sensillum types than s. chaetica.

\section{Projection Pathway of the Sensory Neurons of LPO in the Central Nervous System}

The finding of stained LPO axons entering the ipsilateral side of the gnathal ganglion via the labial nerve and projecting to three distinct areas, the LPOG, gnathal ganglion, and proTG, is similar to the projection pattern previously reported in Helicoverpa armigera and other species (Bogner et al., 1986; Kent et al., 1986; Zhao et al., 2013). Several studies on moths have demonstrated that the sensory neurons in the LPO respond to $\mathrm{CO}_{2}$ (Bogner, 1990; Stange, 1992; Stange et al., 1995; Guerenstein et al., 2004). It is therefore reasonable to assume that the LPO neurons of $M y$. separata are involved in $\mathrm{CO}_{2}$ detection as well. In particular, it would be interesting to investigate whether this kind of signaling might be involved in the long-distance migration of My. separata, taking place at altitudes of $200-500 \mathrm{~m}$ (Zhang et al., 2013).

The staining pattern in the LPOG of $M y$. separata, including terminals from LPO axons filling the whole glomerulus, and no antennal axons, is in full agreement with previous reports in other moth species (Kent et al., 1986; Bogner, 1990; Zhao et al., 2013). Correspondingly, in the mosquitos, Ae. aegypti, and An. gambiae, $\mathrm{CO}_{2}$ sensitive neurons located on the distal segment of the maxillary palps project to AL glomeruli that receive no input from antennal sensory neurons (Distler and Boeckh, 1997; Anton et al., 2003). The finding of a few processes extending outside of LPOG in My. separata is not reported in any of the other studied species.

As mentioned above, the target regions of LPO projections in the gnathal ganglion and proTG of $M y$. separata, were also observed in Helicoverpa armigera (Zhao et al., 2013). Also, previous findings from these two moth species have reported two morphologically corresponding types of LPO sensilla, one hair-shaped and one club-shaped (Zhao et al., 2013; Dong et al., 2014). In future studies, it would be interesting to map the projection pattern of axons originating from each sensillum category.

\section{CONCLUSION}

The results of the present study show that the axons projecting from two sensory appendages of the moth My. separata, i.e., the antenna and the LPO, target multiple neuropils in the central nervous system including the protocerebrum, AL, AMMC, gnathal ganglion, and thoracic ganglia. These findings suggest that the antennae and LPOs play multiple roles in mediating insect behaviors. The AL is the major target for sensory axons originating from both sensory appendages. Furthermore, the AMMC, which is the second major target area for the antennal axons, receives no input from the LPO. The spatial organization of the terminals into segregated zones, shown for the first time in moths, indicates that the function of the mechanosensory neurons differs. The finding of processes from both the antenna and LPO in the gnathal ganglion suggests their roles in detection of gustation-related cues. In the ventral nerve cord, the processes from the LPO sensory neurons terminate in the proTG only, while the antennal afferents target both the proTG and mesoTG. Taken together, the results presented here provide a map of central projections originating from two sensory appendages, which might contribute to further understanding of how sensory information is processed and integrated in the central nervous system. This approach is particularly relevant since $M y$. separata is a polyphagous and migratory pest relying on olfactory cues to locate nectar as well as mates, and probably integrating the chemosensory input with signals from other modalities during long-distance flight behavior.

\section{AUTHOR CONTRIBUTIONS}

Study concept and design: B-WM and X-CZ. Acquisition of data: B-WM, X-CZ, G-YX, and Q-BT. Analysis and interpretation of data: B-WM and X-CZ. Drafting of the manuscript: $\mathrm{X}-\mathrm{CZ}$ and BB. Final manuscript: X-CZ, BB, and G-RW. Obtained funding: $\mathrm{X}-\mathrm{CZ}$ and G-RW. 


\section{FUNDING}

National Natural Science Foundation of China (Nos. 31471830; 31230062). Henan Academician Workstation of Pest Green Prevention and Control for Plants in Southern Henan (Y201504).

\section{REFERENCES}

Ai, H., Nishino, H., and Itoh, T. (2007). Topographic organization of sensory afferents of Johnston's organ in the honeybee brain. J. Comp. Neurol. 502, 1030-1046. doi: 10.1002/cne.21341

Altner, H., and Loftus, R. (1985). Ultrastructure and function of insect thermo- and hygroreceptors. Annu. Rev. Entomol. 30, 273-295. doi: 10.1146/annurev.en.30. 010185.001421

Anton, S., van Loon, J. J. A., Meijerink, J., Smid, H. M., Takker, M., and Rospars, J. P. (2003). Central projections of olfactory neurons from single antennal and palpal sensilla in mosquitoes. Arthropod Struct. Dev. 32, 319-327. doi: 10.1016/ j.asd.2003.09.002

Barrozo, R., Couton, L., Lazzari, C. R., Insausti, T., Minoli, S. A., Fresquet, N., et al. (2009). Antennal pathways in the central nervous system of a blook-sucking bug, Rhodnius prolixus. Arthropod Struct. Dev. 38, 101-110. doi: 10.1016/j.asd. 2008.08.004

Bogner, F. (1990). Sensory physiological investigation of carbon dioxide receptors in lepidoptera. J. Insect Physiol. 36, 951-957. doi: 10.1016/0022-1910(90) 90083-R

Bogner, F., Boppré, M., Ernst, K. D., and Boeckh, J. (1986). CO 2 sensitive receptors on labial palps of Rhodogastria moths (Lepidoptera: Arctiidae): physiology, fine structure and central projection. J. Comp. Physiol. A 158, 741-749. doi: 10.1007/BF01324818

Bräunig, P., Pflüger, H. J., and Hustert, R. (1983). The specificity of central nervous projections of locust mechanoreceptors. J. Comp. Neurol. 218, 197-207. doi: $10.1002 /$ cne.902180207

Chang, X. Q., Zhang, S., Lv, L., and Wang, M. Q. (2015). Insight into the ultrastructure of antennal sensilla of Mythimna separata (Lepidoptera: Noctuidae). J. Insect Sci. 15, 124. doi: 10.1093/jises/iev103

Chen, R. L., Bao, X. Z., Drake, V. A., Farrow, R. A., Wang, S. Y., Sun, Y. J., et al. (1989). Radar observations of the spring migration into northeastern China of the oriental armyworm moth, Mythimna separata, and other insects. Ecol. Entomol. 14, 149-162. doi: 10.1111/j.1365-2311.1989.tb00765.x

Christensen, T. A., and Hildebrand, J. G. (2002). Pheromonal and host-odor processing in the insect antennal lobe: how different? Curr. Opin. Neurobiol. 12, 393-399. doi: 10.1016/S0959-4388(02)00336-7

Crespo, J. G., and Vickers, N. J. (2012). Antennal lobe organization in the slender pigeon louse, Columbicola columbae (Phthiraptera: Ischnocera). Arthropod Struct. Dev. 41, 227-230. doi: 10.1016/j.asd.2012.02.008

de Bruyne, M., Foster, K., and Carlson, J. R. (2001). Odor coding in the Drosophila antenna. Neuron 30, 537-552. doi: 10.1016/S0896-6273(01)00 289-6

Dieudonné, A., Dandiel, T. L., and Sane, S. P. (2014). Encoding properties of the mechanosensory neurons in the Johnston's organ of the hawk moth, Manduca sexta. J. Exp. Biol. 217, 3045-3056. doi: 10.1242/jeb.101568

Dippel, S., Kollmann, M., Oberhofer, G., Montino, A., Krala, M., Rexer, K. H., et al. (2016). Morphological and transcriptomic analysis of a beetle chemosensory system reveals a gnathal olfactory center. BMC Biol. 14:90. doi: 10.1186/s12915016-0304-z

Distler, P. G., and Boeckh, J. (1997). Central projections of the maxillary and antennal nerves in the mosquito Aedes aegypti. J. Exp. Biol. 200, 1873-1879.

Dong, J. F., Liu, H., Tang, Q. B., Liu, Y., Zhao, X. C., and Wang, G. R. (2014). Morphology, type and distribution of the labial-palp pit organ and its sensilla in the oriental armyworm, Mythimna separata (Lepidoptera: Noctuidae). Acta Entomol. Sin. 57, 681-687.

Enjin, A., Zaharieva, E. E., Frank, D. D., Mansourian, S., Suh, G. S. B., Gallio, M., et al. (2016). Humidity sensing in Drosophila. Curr. Biol. 26, 1352-1358. doi: 10.1016/j.cub.2016.03.049

\section{ACKNOWLEDGMENT}

The authors are grateful to Xiang-Zhen Xiao (Henan Institute of Science and Technology) for help with the laser scanning confocal microscopy.

Feng, H. Q., Zhao, X. C., Wu, X. F., Wu, B., Wu, K. M., Cheng, D. F., et al. (2008). Autumn migration of Mythimna separata (Lepidoptera: Noctuidae) over the Bohai sea in Northern China. Environ. Entomol. 37, 774-781. doi: 10.1093/ee/ 37.3.774

Frank, D. D., Enjin, A., Jouandet, G. C., Zaharieva, E. E., Para, A., Stensmyr, M. C., et al. (2017). Early integration of temperature and humidity stimuli in the Drosophila brain. Curr. Biol. 27, 2381-2388. doi: 10.1016/j.cub.2017.06.077

Gao, Q., Yuan, B., and Chess, A. (2000). Convergent projections of the Drosophila olfactory neurons to specific glomeruli in the antennal lobe. Nat. Neurosci. 3, 780-785. doi: $10.1038 / 75753$

Guerenstein, P. G., and Hildebrand, J. G. (2008). Roles and effects of environmental carbon dioxide in insect life. Annu. Rev. Entomol. 53, 161-178. doi: 10.1146/ annurev.ento.53.103106.093402

Guerenstein, P. G., Yepez, E. A., van Haren, J., Williams, D. G., and Hildebrand, J. G. (2004). Floral $\mathrm{CO}_{2}$ emission may indicate food abundance to nectarfeeding moths. Naturwissenschaften 91, 329-333. doi: 10.1007/s00114-0040532- $\mathrm{x}$

Guerra, P. A., Gegear, R. J., and Reppert, S. M. (2014). A magnetic compass aids monarch butterfly migration. Nat. Commun. 5, 4164. doi: 10.1038/ncomms5164

He, Y., Feng, B., Guo, Q., and Du, Y. (2017). Age influences the olfactory profiles of the migratory oriental armyworm Mythimna separata at the molecular level. BMC Genomics 18:32. doi: 10.1186/s12864-016-3427-2

Ignell, R., Dekker, T., Ghaninia, M., and Hansson, B. S. (2005). Neuronal architecture of the mosquito deutocerebrum. J. Comp. Neurol. 493, 207-240. doi: $10.1002 / \mathrm{cne} .20800$

Jiang, Y. Y., Li, C. G., Zeng, J., and Liu, J. (2014). Population dynamics of the armyworm in China: a review of the past 60 years' research. Chin. J. Appl. Entomol. 51, 890-898.

Jørgensen, K., Kvello, P., Almaas, T. J., and Mustaparta, H. (2006). Two closely located areas in the suboesophageal ganglion and the tritocerebrum receive projections of gustatory receptor neurons located on the antennae and the proboscis in the moth Heliothis virescens. J. Comp. Neurol. 496, 121-134. doi: $10.1002 /$ cne. 20908

Kamikouchi, A., Inagaki, H. K., Effertz, T., Hendrich, O., Fiala, A., Göpfert, M. C., et al. (2009). The neural basis of Drosophila gravity-sensing and hearing. Nature 458, 165-171. doi: 10.1038/nature07810

Kamikouchi, A., Shimada, T., and Ito, K. (2006). Comprehensive classification of the auditory sensory projections in the brain of the fruit fly Drosophila melanogaster. J. Comp. Neurol. 499, 317-356. doi: 10.1002/cne.21075

Keil, T. A. (1999). "Morphology and development of the peripheral olfactory organs," in Insect Olfaction, ed. B. S. Hansson (Berlin: Springer), 5-47. doi: 10.1007/978-3-662-07911-9_2

Kent, K. S., Harrow, I. D., Quartararo, P., and Hildebrand, J. G. (1986). An accessory olfactory pathway in Lepidoptera: the labial pit organ and its central projections in Manduca sexta and certain other sphinx moths and silk moths. Cell Tissue Res. 245, 237-245. doi: 10.1007/BF00213927

Kloppenburg, P., Camazine, S. M., Sun, X. J., Randolph, P., and Hildebrand, J. G. (1997). Organization of the antennal motor system in the sphinx moth Manduca sexta. Cell Tissue Res. 287, 425-433. doi: 10.1007/s004410050767

Kollmann, M., Minoli, S., Bonhomme, J., Homberg, U., Schachtner, J., Tagu, D., et al. (2011). Revisiting the anatomy of the central nervous system of a hemimetabolous model insect species: the pea aphid Acyrthosiphon pisum. Cell Tissue Res. 343, 343-355. doi: 10.1007/s00441-010-1099-9

Krishnan, A., Prabhakar, S., Sudarsan, S., and Sane, S. P. (2012). The neural mechanisms of antennal positioning in flying moths. J. Exp. Biol. 215, 3096-3105. doi: 10.1242/jeb.071704

Krishnan, A., and Sane, S. P. (2015). Antennal mechanosensors and their evolutionary antecedents. Adv. Insect Physiol. 49, 59-99. doi: 10.1016/bs.aiip. 2015.06.003 
Kristoffersen, L., Hansson, B. S., Anderbrant, O., and Larsson, M. C. (2008). Aglomerular hemipteran antennal lobes-basic neuroanatomy of a small nose. Chem. Senses 33, 771-778. doi: 10.1093/chemse/bjn044

Lin, C. S. (1990). The Physiology and Ecology of the Oriental Armyworm. Beijing: Peking University Press.

Merlin, C., Gegear, R. J., and Reppert, S. M. (2009). Antennal circadian clocks coordinate sun compass orientation in migratory monarch butterflies. Science 325, 1700-1704. doi: 10.1126/science.1176221

Nässel, D. R., Högmo, O., and Hallberg, E. (1984). Antennal receptors in the blowfly Calliphora erythrocephala. I. The gigantic central projection of the pedicellar campaniform sensillum. J. Morphol. 180, 159-169. doi: 10.1002/jmor. 1051800206

Nishikawa, M., Yokohari, F., and Ishibashi, T. (1995). Central projections of the antennal cold receptor neurons and hygroreceptor neurons of the cockroach Periplaneta americana. J. Comp. Neurol. 361, 165-176. doi: 10.1002/cne. 903610113

Nishino, H., Nishikawa, M., Mizunami, M., and Yokohari, F. (2009). Functional and topographic segregation of glomeruli revealed by local staining of antennal sensory neurons in the honeybee Apis mellifera. J. Comp. Neurol. 515, 161-180. doi: 10.1002/cne.22064

Nishino, H., Nishikawa, M., Yokohari, F., and Mizunami, M. (2005). Dual, multilayered somatosensory maps formed by antennal tactile and contact chemosensory afferents in an insect brain. J. Comp. Neurol. 493, 291-308. doi: 10.1002/cne.20757

Popescu, A., Couton, L., Almaas, T. J., Rospars, J. P., Wright, G. A., Marion-Poll, F., et al. (2013). Function and central projections of gustatory receptor neurons on the antenna of the noctuid moth Spodoptera littoralis. J. Comp. Physiol. A 199, 403-416. doi: 10.1007/s00359-013-0803-0

Rebora, M., Dell'Otto, A., Rybak, J., Piersanti, S., Gaino, E., and Hansson, B. S. (2013). The antennal lobe of Libellula depressa (Odonata, Libellulidae). Zoology 116, 205-214. doi: 10.1016/j.zool.2013.04.001

Sane, S. P., Dieudonné, A., Willis, M. A., and Daniel, T. L. (2007). Antennal mechanosensors mediate flight control in moths. Science 315, 863-866. doi: 10.1126/science. 1133598

Schneider, D. (1964). Insect antennae. Annu. Rev. Entomol. 9, 103-122. doi: 10.1146/annurev.en.09.010164.000535

Stange, G. (1992). High resolution measurement of atmospheric carbon dioxide concentration changes by the labial palp organ of the moth Heliothis armigera (Lepidoptera: Noctuidae). J. Comp. Physiol. A 171, 317-324. doi: 10.1007/ BF00223962

Stange, G. (1997). Effects of changes in atmospheric carbon dioxide on the location of hosts by the moth, Cactoblanstis cactorum. Oecologia 110, 539-545. doi: $10.1007 /$ s004420050192

Stange, G., Monro, J., Stowe, S., and Osmond, C. B. (1995). The $\mathrm{CO}_{2}$ sense of the moth Cactoblastis cactorum and its probable role in the biological control of the CAM plant Opuntia stricta. Oecologia 102, 341-352. doi: 10.1007/BF00329801
Stocker, R. F., Singh, R. N., Schorderet, M., and Siddiqi, O. (1983). Projection patterns of different types of antennal sensilla in the antennal glomeruli of Drosophila melanogaster. Cell Tissue Res. 232, 237-248. doi: 10.1007/ BF00213783

Vosshall, L. B., Wong, A. B., and Axel, R. (2000). An olfactory sensory map in the fly brain. Cell 21, 147-159. doi: 10.1016/S0092-8674(00)00021-0

Xie, G. Y., Zhao, X. C., Ma, B. W., Guo, P., Li, G. P., Feng, H. Q., et al. (2016). Central projection of antennal sensory neurons in the central nervous system of the mirid bug Apolygus lucorum (Meyer-Dür). PLOS ONE 11:e0160161. doi: 10.1371/journal.pone.0160161

Xu, J., Pan, W., Zhang, Y., Li, Y., Wan, G., Chen, F., et al. (2017). Behavioral evidence for a magnetic sense in the oriental armyworm, Mythimna separata. Biol. Open 6, 340-347. doi: 10.1242/bio.022954

Yoritsune, A., and Aonuma, H. (2012). The anatomical pathways for antennal sensory information in the central nervous system of the cricket, Gryllus bimaculatus. Invert. Neurosci. 12, 103-117. doi: 10.1007/s10158-0120137-6

Yorozu, S., Wong, A., Fischer, B. J., Dankert, H., Kernan, M. J., Kamikouchi, A., et al. (2009). Distinct sensory representations of wind and near-field sound in the Drosophila brain. Nature 458, 201-205. doi: 10.1038/nature07843

Zhang, Y. H., Zhang, Z., Li, C., Jiang, Y. Y., Zeng, J., and Cheng, D. F. (2013). Seasonal migratory behavior of Mythimna separata (Lepidoptera: Noctuidae) in northeast China. Acta Entomol. Sin. 56, 1418-1429.

Zhao, X. C., Chen, Q. Y., Guo, P., Xie, G. Y., Tang, Q. B., Guo, X. R., et al. (2016). Glomerular identification in the antennal lobe of the male moth Helicoverpa armigera. J. Comp. Neurol 524, 2993-3013. doi: 10.1002/cne. 24003

Zhao, X. C., Feng, H. G., Wu, B., Wu, X. F., Liu, Z. F., Wu, K. M., et al. (2009). Does the onset of sexual maturation terminate the expression of migratory behavior in moths? A study of the oriental armyworm, Mythimna separata. J. Insect Physiol. 55, 1039-1043. doi: 10.1016/j.jinsphys.2009.07.007

Zhao, X. C., Tang, Q. B., Berg, B. G., Liu, Y., Wang, Y. R., Yang, F. M., et al. (2013). Fine structure and primary sensory projections of sensilla located in the labialpalp pit organ of Helicoverpa armigera (Insecta). Cell Tissue Res. 353, 399-408. doi: 10.1007/s00441-013-1657-z

Conflict of Interest Statement: The authors declare that the research was conducted in the absence of any commercial or financial relationships that could be construed as a potential conflict of interest.

Copyright $\odot 2017$ Ma, Zhao, Berg, Xie, Tang and Wang. This is an open-access article distributed under the terms of the Creative Commons Attribution License (CC BY). The use, distribution or reproduction in other forums is permitted, provided the original author(s) or licensor are credited and that the original publication in this journal is cited, in accordance with accepted academic practice. No use, distribution or reproduction is permitted which does not comply with these terms. 